\title{
Configurações
}

Revista Ciências Sociais

\section{Fornecer leituras sociológicas da realidade: prática pedagógica da sociologia no ensino de não sociólogos}

Providing sociological readings of reality: pedagogical practice of sociology in teaching non-sociologists

offrir des lectures sociologiques du réel : pratique pédagogique de la sociologie dans l'enseignement des non-sociologues

\section{SANDRA GOMES}

\section{OpenEdition}

Journals

Edição electrónica

URL: https://journals.openedition.org/configuracoes/14607

DOI: 10.4000/configuracoes. 14607

ISSN: 2182-7419

Editora

Centro de Investigação em Ciências Sociais

Edição impressa

Paginação: 123-138

ISSN: 1646-5075

\section{Refêrencia eletrónica}

SANDRA GOMES, «Fornecer leituras sociológicas da realidade: prática pedagógica da sociologia no ensino de não sociólogos», Configurações [Online], 28 | 2021, posto online no dia 28 dezembro 2021 , consultado o 30 dezembro 2021. URL: http://journals.openedition.org/configuracoes/14607 ; DOI: https://doi.org/10.4000/configuracoes. 14607 
GOMES, Sandra - Fornecer leituras sociológicas da realidade: prática pedagógica da sociologia no ensino de não sociólogos. Configurações [em linha]. 28 (2021) p. 123-138.

\title{
Fornecer leituras sociológicas da realidade: prática pedagógica da sociologia no ensino de não sociólogos
}

SANDRA GOMES *

IPAM- Instituto Português de Administração de Marketing do Porto

\begin{abstract}
Resumo
O presente artigo relata uma ex periência pedagógica de ensino de uma área de conhecimento interdisciplinar, Comportamento Organizacional, lecionada por uma socióloga a estudantes não sociólogos. Procurou-se refletir sobre a forma como a aprendizagem colaborativa pode potenciar a apreensão e aplicação de conhecimentos no ensino superior. A responsabilidade social das organizações foi o tema escolhido para a exemplificação do caso em estudo. Foi realizado um inquérito aos estudantes antes do início da lecionação dos conteúdos e após a conclusão dos mesmos. Os estudantes valorizaram de forma positiva essencialmente a possibilidade de aplicação dos conhecimentos a um problema real de uma organização.
\end{abstract}

Palavras-chave: prática pedagógica, sociologia, responsabilidade social das organizações, aprendizagem colaborativa.

\begin{abstract}
Providing sociological readings of reality: pedagogical practice of sociology in teaching non-sociologists

This article reports on a pedagogical teaching experience in an interdisciplinary area of knowledge, Organisational Behaviour, taught by a sociologist to non-sociologist students. We sought to reflect on how collaborative learning can enhance the acquisition and application of knowledge in higher education. The social responsibility of organisations was the theme chosen to illustrate the case under study. A survey was carried out before the beginning of the teaching experience and after its completion. Students positively valued the application of knowledge to a real organisational problem.
\end{abstract}

*E-mail: sgomes@ipam.pt | ORCID ID: https://orcid.org/0000-0002-5987-1631 
Keywords: pedagogical practice, sociology, social responsibility of organisations, collaborative learning.

\section{Resumé \\ Offrir des lectures sociologiques du réel : pratique pédagogique de la sociologie dans l'enseignement des non-sociologues}

Cet article rend compte d'une expérience d'enseignement pédagogique dans un domaine interdisciplinaire de connaissances - Comportement organisationnel - enseignée par une sociologue à un groupe d'étudiants non-sociologues. Nous avons cherché à réfléchir à la manière dont l'apprentissage collaboratif peut améliorer l'acquisition et l'application des connaissances chez les étudiants de l'enseignement supérieur. La responsabilité sociale des organisations a été le thème choisi pour illustrer le cas à l'étude. Une enquête auprès des étudiants a été réalisée avant le début de l'enseignement des contenus et après son achèvement. Les étudiants ont évalué positivement l'application des connaissances à un problème réel dans une organisation.

Mots-clés: pratique pédagogique, sociologie, responsabilité sociale des organisations, apprentissage collaboratif.

\section{Introdução}

Este artigo pretende dar conta de uma experiência pedagógica desenvolvida por uma socióloga no exercício da sua função de docência numa instituição que forma futuros profissionais na área da gestão. Não se pretende elaborar um ensaio teórico sobre a prática pedagógica do ensino superior de sociologia, mas sim partilhar de forma transparente uma experiência pessoal e pedagógica concreta.

Esta experiência pedagógica situa-se no âmbito de uma unidade curricular interdisciplinar, não especificamente de sociologia, mas que sendo lecionada por uma socióloga incorpora uma perspetiva sociológica na sua abordagem. A unidade curricular em questão é Comportamento Organizacional e o tema específico que aqui é tratado versa a Responsabilidade Social das Organizações (RSO).

Existem vários estudos prévios que abordam experiências pedagógicas de ensino de sociologia em escolas de gestão (Duarte, 2009; Vidal, 2010; Baecker, 2016), contudo existe alguma escassez no que respeita à experiência de sociólogos em práticas pedagógicas aplicadas a áreas do conhecimento mais interdisciplinares.

O ensino de sociologia direcionado a futuros não sociólogos configura algumas particularidades e alguns desafios adicionais (Gomes, 2004). A Sociologia pode ajudar a formar melhores gestores, capazes de pensamento 
crítico e de um olhar para a realidade social de forma reflexiva, desenvolvendo “explicações para um mundo já explicado" (Giddens, 1992).

Ir-se-á fazer um enquadramento da experiência e da prática pedagógica, procurando intercalar com breves referências de sustentação teórica. Este tex to não pretende ter a estrutura tipo de um artigo académico tradicional. Tem, assim, como objetivo apresentar um relato de uma experiência pedagógica que pelos resultados obtidos acredita-se poder ser replicado em outros contextos. Poderá funcionar também como um alerta de que este tema deve entrar seriamente na agenda dos sociólogos de forma a existir um olhar sociológico sobre a RSO, quer ao nível da investigação, quer do ensino.

Este artigo subdivide-se em três partes: inicialmente faz-se um enquadramento do contexto onde decorre a experiência pedagógica; na segunda parte procede-se a uma descrição da experiência pedagógica, do planeamento das sessões e dos instrumentos pedagógicos utilizados, enquadrados num quadro de aprendizagem colaborativa; por fim apresentam-se os resultados dos inquéritos realizados aos estudantes: o primeiro inquérito realizado antes das sessões em que se avaliou o grau de conhecimento e sensibilidade para o tema da responsabilidade social organizacional, e o segundo inquérito, pós-módulo, destinava-se a avaliar o desenvolvimento dos conhecimentos, bem como a própria experiência pedagógica utilizada.

De acordo com o Primeiro Inquérito às Práticas Profissionais dos Diplomados em Sociologia (APS, 2013), pelo menos 40\% dos sociólogos desenvolvia a sua atividade no setor da educação e investigação, pelo que importa partilhar experiências pedagógicas variadas para que se possa replicar potenciais boas práticas.

\section{Relato de uma experiência na primeira pessoa}

"A primeira tarefa do professor de sociologia é fazer com que os alunos questionem o que eles normalmente consideram garantido" (Laz, 2020, p. 57).

Ensinar é um ato político (Apple, 2006), e como tal importa enquadrar o contex to histórico e social. Não é indiferente praticar o ensino de sociologia em instituições que formem ou não sociólogos.

$\mathrm{Na}$ instituição objeto da experiência pessoal aqui relatada, a sociologia foi perdendo lugar de destaque e passou a viver escondida com nomes mais abrangentes mas que, com a manutenção de docentes com formação em sociologia, se foi impondo através de outras máscaras ${ }^{1}$. O olhar sociológico e os conteúdos 
que visam conhecimento sociológico existem e afirmam-se em unidades curriculares distintas, infelizmente nenhuma delas com o nome de sociologia.

Poder-se-ia abordar neste contexto várias práticas pedagógicas em diferentes unidades curriculares nesta instituição, algumas delas bem mais relacionadas explicitamente com a sociologia enquanto campo do saber. Exemplos como a diversidade cultural, o relativismo cultural, a socialização de género, os modelos de caracterização das culturas, o consumo simbólico, a estrutura de classes e o seu impacto no consumo, são temas bastante trabalhados e debatidos nesta instituição na formação de futuros profissionais.

A lecionar há mais de 20 anos na mesma instituição o percurso da autora foi repleto de aprendizagens. Iniciou-se com a constatação de um choque cultural inicial entre a cultura sociológica existente, decorrente do processo de socialização da licenciatura e mestrado na área científica da Sociologia, e a cultura de uma escola na área da gestão. Durante algum tempo o sentimento era o de fazer parte de um corpo estranho, sentimento partilhado com outros sociólogos em escolas de gestão noutros contextos culturais (Duarte, 2009).

A Sociologia como campo do saber em território de formação de gestores foi-se impondo e foi construindo a sua própria identidade. Foi necessário muito trabalho interdisciplinar e muita aprendizagem de saberes da área da gestão para que a Sociologia se fosse afirmando como uma ciência social que, efetivamente, pode ajudar a construir significados e desenvolver abordagens sistémicas, compreensivas e úteis (já para não dizer imprescindíveis) ao trabalho de um gestor de marketing. À medida que ia aumentando o conhecimento sobre a área científica de acolhimento, mais facilmente foi possível falar de Sociologia a partir das suas abordagens, dos seus problemas e das suas necessidades. E a voz da sociologia foi-se conseguindo impor e ouvir. Não deixando de ser uma socióloga, com uma "bagagem epistemológica" (Duarte, 2009) própria a fazer leituras sociológicas nesta aproximação às ciências empresariais, esta tornou-se útil na sala de aula e irá certamente ser fundamental no mercado de trabalho e no exercício da cidadania dos estudantes.

\section{Enquadramento da prática pedagógica}

A prática pedagógica que é partilhada neste contexto enquadra-se numa Unidade Curricular (UC) denominada Comportamento Organizacional, lecionada numa instituição privada portuguesa de ensino superior politécnico. O módulo em questão denomina-se Ética e Responsabilidade Social nas Organizações. Não sendo uma UC, nem um módulo, especificamente de Sociologia, é ministrada por uma socióloga, usando as ferramentas teóricas e metodológicas da Sociologia.

Esta UC é dividida em aulas teóricas (1 hora semanal), meramente expositivas e interrogativas sobre os conteúdos teóricos programados, e em aulas 
teórico-práticas ( 2 horas semanais), em que se pressupõe um trabalho mais aplicado à realidade e que permita uma maior concretização dos conteúdos lecionados previamente.

Estando enquadrada na área científica de Ciências Sociais e Humanas, esta UC pretende que os estudantes construam uma compreensão deste fenómeno social total - a Responsabilidade Social das Organizações (RSO) - que rompa com alguns apriorismos decorrentes do senso comum e os faça refletir criticamente sobre a realidade social.

Existem alguns artigos que apontaram previamente instrumentos pedagógicos para o ensino do tema da RSO em cursos de gestão (Rendtorff, 2015; Brinkmann, 2019), contudo esta área do conhecimento permanece ainda subinvestigada.

De acordo com Blanco (2010), existe uma escassez de investigação na área da sociologia sobre o tema da responsabilidade social. Que conhecimento difundem os sociólogos sobre RSO? Este autor concluiu que existia "um tímido interesse despertado para a responsabilidade social das empresas dentro do âmbito específico da sociologia" (Blanco, 2010, p. 590). Segundo o mesmo autor, a Sociologia como ciência pode/deve realizar importantes contribuições a três níveis para um melhor conhecimento sobre responsabilidade social das empresas: nível teórico (investigação sobre RSO), aplicado (colaboração no desenho de planos estratégicos e operacionais de RSO) e institucional através das suas representações académicas e institucionais (elaboração de propostas e normas) (Blanco, 2010, p. 590). Nesta UC em particular são trabalhados os níveis teórico e aplicado, na medida em que os estudantes têm de desenvolver um conhecimento mais aprofundado sobre o tema e devem aplicá-lo a uma organização (através de um diagnóstico e plano de ação).

Foi esta a motivação para a escolha desta UC, e deste módulo em concreto, para partilha neste artigo. Poderá funcionar também como um alerta de que este tema deve entrar seriamente na agenda dos sociólogos de forma a existir um olhar sociológico sobre a RSO, quer ao nível da investigação, quer do ensino.

De acordo com as Nações Unidas, uma interessante matriz de princípios, envolvendo três abordagens, deve ser considerada no desenvolvimento de conteúdos com foco na RSO: uma perspetiva macro do conhecimento do desenvolvimento sustentável; uma perspetiva meso de responsabilidade organizacional, segundo a qual todas as organizações têm a responsabilidade de contribuir para o desenvolvimento sustentável; e, finalmente, uma perspetiva micro de responsabilidade pessoal, em que os indivíduos têm a responsabilidade de contribuir para o desenvolvimento sustentável (United Nations, 2015). Estes princípios foram tidos em conta na construção dos conteúdos e na sua operacionalização nesta UC. 
Nas aulas teóricas foram abordados os seguintes conteúdos: conceito de responsabilidade social e de desenvolvimento sustentável; as dimensões da responsabilidade social; contexto socio-histórico de surgimento e desenvolvimento do tema e tendências futuras; algumas referências de enquadramento; os objetivos de desenvolvimento sustentável das Nações Unidas, Global Compact, SA8000, ISO 26000; metodologia de envolvimento de stakeholders e a importância das redes de parceria; e, por fim, a gestão da ética nas organizações.

Este conteúdo programático é avaliado no teste de avaliação e igualmente no desenvolvimento de um trabalho prático de grupo, realizado em dois meses do semestre. Este trabalho traduz-se na realização de um diagnóstico à responsabilidade social de uma empresa e no desenvolvimento do respetivo plano de intervenção.

Tal como defendido previamente por vários autores (Pedersen, 2010), os estudantes valorizam aprendizagens que façam uma aplicação da teoria à realidade. Muitos estudantes têm manifestado alguma frustração com a teoria quando esta se mantém unicamente num plano mais abstrato. A aplicação de ferramentas de aprendizagem ativa (active learning) no ensino superior, assente numa perspetiva construtivista sobre a aquisição de conhecimento, não sendo um tema novo, tem revelado excelentes resultados. Alguns estudos têm sido publicados com a aplicação da aprendizagem ativa e colaborativa à área científica de sociologia (Mayberry, 1998; Pederson, 2010; Tolich et al., 2017). De acordo com Pederson (2010), a aprendizagem colaborativa, com a sua relação com a aprendizagem ativa, torna-se um "caminho promissor para o ensino da teoria sociológica".

As abordagens centradas na aprendizagem colaborativa (collaborative learning) e aprendizagem baseada em projetos (project based learning) reestruturam o ambiente educativo no qual os estudantes aprendem. A aprendizagem centra-se no trabalho de grupo, definição de problemas, pesquisa de informação, interpretação de resultados e partilha de conclusões (Pederson, 2010; O’Donnell e H melo-Silver, 2013; Loes e Pascarella, 2017). Acredita-se que esta metodologia fomenta competências importantes nos estudantes, nomeadamente a reflexão, reflexividade e pensamento crítico, o que lhes permite ir para além de uma descrição do fenómeno organizacional (Duarte, 2009).

Já vários anos antes DiTomaso (1986) referia que as experiências pedagógicas experienciais, inicialmente mais prevalecentes nas escolas de gestão do que nas de sociologia, eram um fator potenciador de um maior nível de satisfação dos estudantes.

O trabalho de grupo desenvolve, nos estudantes, competências interpessoais fundamentais e responsabiliza-os pela participação nas atividades desenvolvidas nas aulas.

Este trabalho tem uma ponderação na nota final de $25 \%$ e é desenvolvido em grupos constituídos, no máximo, por 5 elementos. Cada turma possui, em 
média, cerca de 6 grupos. Existe atualmente na instituição um total de sete turmas. No momento final de apresentação e defesa dos trabalhos pelos grupos é realizado um exercício de auto e heteroavaliação que permite, juntamente com a avaliação contínua feita pela docente ao longo das sessões de acompanhamento dos trabalhos, enquadrar classificações diferenciadas para cada elemento do grupo.

Em termos dos objetivos deste trabalho, destacam-se: 1. Efetuar um diagnóstico de responsabilidade social da empresa em estudo, a partir dos conteúdos teóricos desenvolvidos em aula; 2. Compreender as especificidades da responsabilidade social no setor em estudo, a partir de pesquisa de artigos científicos sobre o tema; 3 . Identificar, a partir da utilização da ferramenta de benchmarking, as boas práticas de responsabilidade social neste setor, a nível nacional e internacional; 4. Identificar área(s) de intervenção prioritária(s) na empresa em estudo; 5. Elaborar uma proposta de intervenção ao nível de responsabilidade social sustentada no diagnóstico.

A planificação simplificada das sessões práticas encontra-se explicada no quadro seguinte. É fundamental o trabalho desenvolvido pelos grupos com o apoio do docente ao longo das semanas. Após um primeiro trabalho de pesquisa e de recolha de informação sobre o caso em estudo, é importante que os estudantes tenham contacto direto com a organização, que poderá relatar na primeira pessoa as suas práticas ao nível da responsabilidade social e quais os desafios que considera existir. No final, são selecionados alguns grupos para apresentação dos seus trabalhos à organização, sendo que esta elege, depois, o melhor trabalho, sendo entregue um certificado e um pequeno prémio de participação a cada elemento.

\section{Tabela 1 - Planeamento das aulas práticas de apoio ao trabalho}

\begin{tabular}{|c|l|}
\hline Sessão 1 & $\begin{array}{l}\text { Elaboração de um inquérito de avaliação das perceções e conhecimentos prévios sobre } \\
\text { o tema da responsabilidade social. Utilização da ferramenta Mentimeter para } \\
\text { construção de nuvens de palavras de significados. } \\
\text { Apresentação do Guia do trabalho prático e da empresa em estudo. Início dos trabalhos } \\
\text { de grupo. Pesquisa sobre empresa e sobre o setor em questão }\end{array}$ \\
\hline Sessão 2 & $\begin{array}{l}\text { Continuação da pesquisa feita e apoio docente com palavras-chave de apoio à pesquisa } \\
\text { e explicação de algumas particularidades setoriais ao nive1 da responsabilidade social }\end{array}$ \\
\hline Sessão 3 & Seminário com a empresa em questão. Se possível, visita às suas instalações. \\
\hline Sessão 4 & $\begin{array}{l}\text { Explicação da ferramenta de benchmarking. Início do trabalho de pesquisa de boas } \\
\text { práticas de responsabilidade social nacionais e internacionais. Acompanhamento do } \\
\text { docente aos diferentes grupos. Cada grupo elege um porta-voz e expõe as conclusões } \\
\text { do trabalho realizado e das dúvidas tidas pelo grupo até ao momento }\end{array}$ \\
\hline Sessão 5 & $\begin{array}{l}\text { Continuação do trabalho de pesquisa das boas práticas. Eleição das melhores práticas } \\
\text { para construção das tabelas de boas práticas de responsabilidade social. }\end{array}$ \\
\hline Sessão 6 & $\begin{array}{l}\text { Identificação dos pontos a melhorar. Trabalho de acompanhamento dos grupos pelo } \\
\text { docente. Cada grupo elege um porta-voz e expõe as ideias do grupo e das dúvidas } \\
\text { existentes }\end{array}$ \\
\hline Sessão 7 & $\begin{array}{l}\text { Defesa dos trabalhos junto do docente. Inquérito de avaliação de conhecimento pós- } \\
\text { formação. }\end{array}$ \\
\hline Sessão 8 & $\begin{array}{l}\text { Avaliação dos trabalhos e envio do respetivo feedback aos estudantes. Seleção dos } \\
\text { melhores trabalhos para apresentação à empresa }\end{array}$ \\
\hline $\begin{array}{l}\text { Apresentação dos melhores trabalhos à empresa e atribuição de prémio e certificado } \\
\text { ao melhor trabalho }\end{array}$ \\
\hline
\end{tabular}


Existem poucos estudos empíricos que investigam a importância de diferentes metodologias para desenvolver as competências de RSO dos estudantes, sob o ponto de vista destes. Com o objetivo de compreender de que forma as ferramentas pedagógicas tiveram impacto no desenvolvimento de competências dos estudantes, foi realizado um estudo que pode ser consultado de forma mais pormenorizada em Gomes et al. (2020). Foram inquiridos 154 estudantes num universo total de 220. A elevada taxa de resposta ficou a dever-se ao facto de os inquéritos terem sido administrados no decurso das aulas da UC em questão.

Os inquéritos foram administrados em dois momentos diferentes: antes e após estes conteúdos terem sido ministrados e trabalhados com os estudantes. O primeiro inquérito destinava-se a aferir o grau de conhecimento dos estudantes sobre RSO previamente à sua experiência letiva e o segundo permitiu fazer a avaliação, quer dos conhecimentos desenvolvidos, quer da própria experiência pedagógica utilizada no decorrer nas sessões.

O primeiro questionário foi realizado antes do início da exposição dos conteúdos, e foi feito em aula presencial, no início de março de 2020. Devido à situação pandémica que surgiu em meados de março 2020, o segundo momento foi adaptado para o ambiente online, após a abordagem do conteúdo teórico e antes da avaliação individual. Esse segundo momento ocorreu em final de abril de 2020 .

O questionário foi composto principalmente por questões abertas, o que permitiu aos estudantes responderem de forma aberta e inspirar a sua autoexpressão e riqueza de detalhes. Além disso, a fim de avaliar o conhecimento dos estudantes, foi pedido que projetassem mapas cognitivos úteis para representar as noções concretas e teóricas sobre RSO, resumindo palavras que os estudantes relacionavam com as diferentes dimensões. O uso de mapas cognitivos é extremamente relevante, por um lado, para avaliar as representações sobre os conceitos e, por outro lado, para avaliar as perceções dos estudantes sobre como organizam o conhecimento nas suas estruturas cognitivas (Lourdel et al., 2007).

As respostas às questões abertas foram codificadas e categorizadas de acordo com os temas emergentes, relacionados com as categorias anteriores abordadas nas aulas teóricas. Os mapas cognitivos, por sua vez, foram explorados por meio de técnicas de análise de conteúdo, utilizando o número de palavras e a qualidade da proposição, ou seja, que palavras ou expressões foram identificadas como corretas, conforme utilizado por alguns autores (Yin et al., 2005). O número e o tipo de palavras associadas a cada dimensão permitem avaliar se as dimensões foram aprendidas corretamente pelos estudantes. 


\section{Apresentação dos resultados de avaliação da experiência pedagógica}

A maioria dos estudantes inquiridos foi do sexo feminino, embora a distribuição por género seja bastante equitativa. Em termos de idade, os estudantes tinham, em média, 20 anos, variando entre 18 e 40 anos. A maioria dos estudantes tinha entre 18 e 20 anos (66\%), e mais de metade possuía algum tipo de experiência profissional.

Tabela 2. Caracterização dos estudantes inquiridos

\begin{tabular}{|c|c|c|c|c|c|c|c|c|}
\hline & \multicolumn{2}{|c|}{ Sexo (\%) } & \multicolumn{4}{|c|}{ Idade (\%) } & \multirow{2}{*}{$\begin{array}{l}\text { Experiência } \\
\text { profissional (\%) }\end{array}$} & \multirow{2}{*}{ Amostra } \\
\hline & $\mathbf{F}$ & M & $18-20$ & $21-25$ & $26-30$ & $30-40$ & & \\
\hline $1^{\circ}$ Inquérito & 53.9 & 46.1 & 66.2 & 14.3 & 4.5 & 3 & 55.8 & 154 \\
\hline $2^{\circ}$ Inquérito & 59.8 & 40.2 & 66.7 & 28.5 & 2.3 & 2.2 & 50.6 & 87 \\
\hline
\end{tabular}

O conhecimento sobre RSO foi avaliado por meio de uma pergunta aberta "O que considera ser a Responsabilidade Social das O rganizações? Explique pelas suas próprias palavras, desenvolvendo o máximo que conseguir”.

O primeiro inquérito revelou que a maioria dos estudantes está essencialmente focada na dimensão social interna e externa da RSO e cerca de $29 \%$ referem aspetos relacionados com a dimensão ambiental. Sublinhe-se que 4,5\% dos estudantes não conseguiram dar qualquer tipo de definição.

Tabela 3. Conhecimento sobre RSO (\% de referências)

\begin{tabular}{|l|c|c|c|}
\hline & $\mathbf{1}^{\mathbf{0}}$ Inquérito (antes) & $\mathbf{2}^{\circ}$ Inquérito (após) & Variação \\
\hline Dimensão Económica & 8.4 & 39.1 & $\mathbf{+ 3 0 . 7}$ \\
\hline Dimensão Social Interna & 53.9 & 86.2 & $\mathbf{+ 3 2 . 3}$ \\
\hline Dimensão Social Externa & 66.9 & 86.2 & +19.3 \\
\hline Dimensão Ambiental & 29.2 & 51.7 & +22.5 \\
\hline Visão estratégica da RSO & 0 & 13.8 & +13.8 \\
\hline Gestão de impactos negativos & 2.6 & 4.6 & +2 \\
\hline Não faz ideia & 4.5 & 3.4 & -1.1 \\
\hline
\end{tabular}

Após a frequência do módulo de RSO, uma percentagem significativa de estudantes conseguiu fazer referências concretas às diferentes dimensões, com destaque para a dimensão social interna e externa (86\%) e também referências à dimensão ambiental (52\%). Em termos de variação, destaca-se o aumento das referências à dimensão social interna $(+32 \%)$ e à dimensão económica (+31\%). Esta análise revela que os estudantes, mesmo sem terem realizado um estudo 
autónomo para a avaliação individual através de uma prova escrita sobre estes conteúdos, apresentam uma melhoria dos seus conhecimentos e uma maior abrangência ligada às categorias definidas no conceito de Responsabilidade Social.

Antes do módulo, apenas $20 \%$ dos estudantes conheciam ou tinham ouvido falar sobre os Objetivos do Desenvolvimento Sustentável das Nações Unidas (ODS) (Agenda 2030). Dos estudantes que relataram saber ou ter ouvido falar, apenas 7\% revelaram, numa questão aberta, conhecimento real sobre os ODS, sendo que $25 \%$ não denotaram ter conhecimento algum. Após a experiência de aprendizagem, 62\% dos estudantes demonstraram saber algo sobre os ODS e deram uma explicação considerando os 17 Objetivos Globais, bem como alguns exemplos de tais objetivos. A percentagem de estudantes que não revelam nenhum conhecimento sobre os ODS diminuiu 16,3\% .

Tabela 4. Conhecimento sobre os ODS (2030)

\begin{tabular}{|l|c|c|c|}
\hline & $\begin{array}{c}\mathbf{1}^{\circ} \text { Inquérito } \\
\text { (antes) }\end{array}$ & $\begin{array}{c}\mathbf{2}^{\mathbf{0}} \text { Inquérito } \\
\text { (após) }\end{array}$ & Variação \\
\hline Definição revela conhecimento & 7,1 & $\mathbf{6 2 . 3}$ & $\mathbf{+ 5 5 . 2}$ \\
\hline Definição revela conhecimento parcial & $\mathbf{6 7 , 9}$ & 29 & -38.9 \\
\hline Definição não revela conhecimento & 25,0 & 8.7 & -16.3 \\
\hline
\end{tabular}

Foram utilizados mapas conceituais para avaliar as representações de RSO dos estudantes em relação às dimensões da RSO. No primeiro inquérito, os estudantes foram solicitados a desenhar palavras relacionadas com cada dimensão numa folha de papel fornecida pela docente. No segundo inquérito, como foi administrado online pelas razões explicadas acima, os estudantes tiveram de preencher os mapas usando o computador, ou desenhar à mão e fazer o upload do ficheiro. As Figuras 1 e 2 exemplificam dois mapas conceituais no primeiro e no segundo momento. 
Figura 1 Exemplo de mapa conceptual $-1^{\circ}$ inquérito

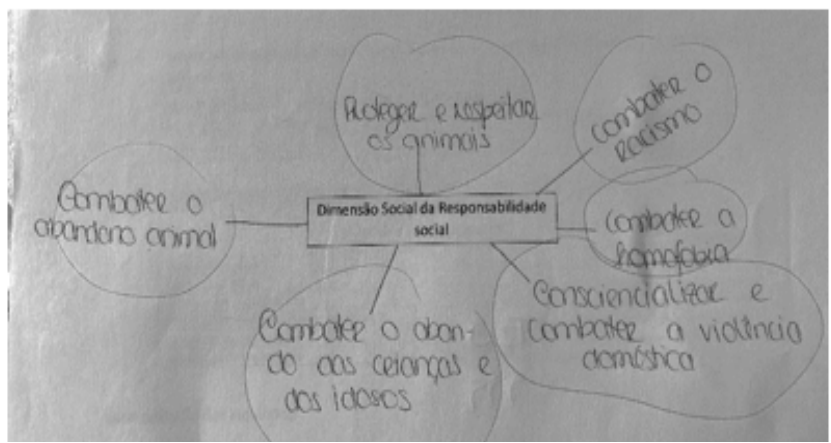

Figura 2. Exemplo de mapa conceptual $-2^{\circ}$ inquérito

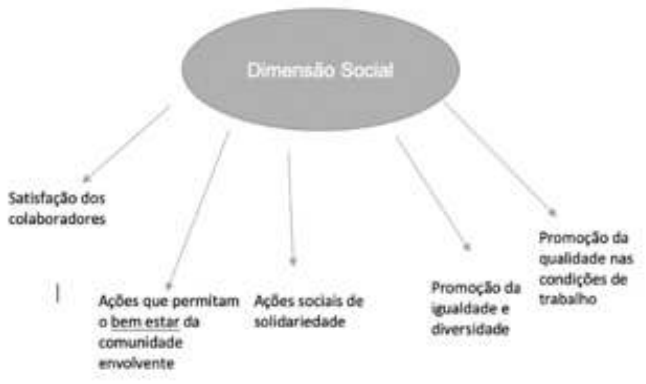

Conforme explicado acima, a análise dos mapas cognitivos foi desenvolvida a partir da quantidade de conceitos que os estudantes conseguiram identificar e a sua correção. A análise do número de palavras nessas categorias permite verificar se o conceito de RSO foi bem compreendido pelos estudantes e traduzido em ideias mais concretas. Os estudantes em ambos os inquéritos revelaram mais dificuldades em associar palavras à dimensão económica. No primeiro inquérito, $20 \%$ a $26 \%$ dos estudantes não conseguiram associar nenhuma palavra a nenhuma das dimensões de RSO. A dimensão em que os estudantes parecem adicionar mais palavras foi a dimensão social, seguida da dimensão ambiental e, por último, a dimensão económica. O número médio de palavras relacionadas é bastante baixo (entre 2,6 e 2,3). A dimensão económica, com menor número de associações de palavras, é também a que apresenta o menor número de palavras corretamente associadas.

Analisando o efeito das experiências formativas nos mapas cognitivos dos estudantes, verificamos que as palavras associadas a todas as dimensões efetivamente aumentaram. A percentagem de estudantes que não conseguiram 
associar palavras às dimensões diminuiu consideravelmente - entre 10,5 e $8,1 \%$.

Tabela 5. Análise de mapas conceptuais

\begin{tabular}{|l|c|c|c|c|c|c|}
\hline & \multicolumn{3}{|c|}{$\mathbf{1}^{\circ}$ Inquérito (antes) } & \multicolumn{2}{|c|}{$\mathbf{2}^{\circ}$ Inquérito (após) } \\
\hline & $\begin{array}{c}\text { Total } \\
\text { palavras }\end{array}$ & $\begin{array}{c}\text { Corretas } \\
\text { (Media) }\end{array}$ & $\begin{array}{c}\text { Nenhuma } \\
\text { palavra } \\
\text { (\%) }\end{array}$ & $\begin{array}{c}\text { Total } \\
\text { palavras }\end{array}$ & $\begin{array}{c}\text { Corretas } \\
\text { (Media) }\end{array}$ & $\begin{array}{c}\text { Nenhuma } \\
\text { palavra } \\
\text { (\%) }\end{array}$ \\
\hline Dimensão Social & 2.6 & 2.89 & 25.3 & 4.48 & 4.53 & 8.1 \\
\hline Dimensão ambiental & 2.45 & 2.66 & 20.1 & 3.95 & 4.24 & 8.1 \\
\hline Dimensão Económica & 2.33 & 1.74 & 26 & 3.47 & 3.13 & 10.5 \\
\hline
\end{tabular}

As questões abertas avaliaram como os estudantes consideram o seu próprio papel como cidadãos socialmente responsáveis.

Em relação aos comportamentos que estes poderiam ter como cidadãos socialmente responsáveis, o impacto da formação parece ser maior, como era expectável. Os estudantes revelaram ter aumentado o seu leque de possibilidades de se comportarem socialmente, como cidadãos, de forma mais responsável, o que parece ser positivo.

O inquérito pré-módulo realizado destacou a dimensão social, em que muitos estudantes relataram que gostariam de fazer mais trabalho voluntário (43\%), bem como a dimensão ambiental (39\%), com muitos deles a indicarem que poderiam começar a fazer mais reciclagem e usar o carro menos vezes. Sublinhe-se a percentagem de estudantes que referiram não saber como serem mais responsáveis socialmente como cidadãos $(23,4 \%)$. No segundo inquérito, a percentagem de estudantes que afirmam não saber o que fazer desce para 4,6\% (variação de -19\%). Assim, ao que parece, a experiência de aprendizagem abriu o seu "máximo de consciência" para outras formas de exercer o papel de cidadãos em prol do desenvolvimento sustentável, o que é bastante interessante e positivo.

Neste inquérito pós-módulo, as dimensões socio-ambientais também foram mais citadas, com percentagens acima de 50\%. É também interessante notar o aumento das referências a comportamentos relacionados com a dimensão económica $(+14,4 \%)$. Alguns comportamentos associados a esta dimensão lidam com o papel dos estudantes como consumidores mais conscientes.

No inquérito pós-módulo, os estudantes foram convidados a avaliar cada uma das suas experiências de aprendizagem para desenvolver as competências sobre RSO (Tabela 6). Em geral, os estudantes avaliaram todos os itens com médias superiores a 4 (1-5). A experiência de trabalhar com um caso real de empresa foi a mais bem avaliada $(4,82)$.

Tabela 6. Avaliação dos estudantes sobre a importância de cada uma das experiências de ensino para o seu conhecimento sobre RSO 
Tabela 6. Avaliação dos estudantes sobre a importância de cada uma das experiências de ensino para o seu conhecimento sobre RSO

\begin{tabular}{|l|c|}
\hline & M (1-5) \\
\hline Trabalho de grupo sobre um caso real & 4.82 \\
\hline Aulas práticas & 4.72 \\
\hline Aulas teóricas & 4.53 \\
\hline $\begin{array}{l}\text { Sessão de trabalho com a empresa para esclarecimento } \\
\text { de dúvidas }\end{array}$ & 4.18 \\
\hline Seminário com a empresa & 4.16 \\
\hline
\end{tabular}

Uma análise comparativa de médias foi realizada usando o T-TEST. As estudantes do sexo feminino revelam avaliações mais elevadas em geral. Existem diferenças estatisticamente significativas em três das experiências de aprendizagem: 'Importância das aulas práticas' ( $\mathrm{p}=0,045)$, que as estudantes do sexo feminino avaliam mais positivamente $(M=4,81)$ do que os estudantes do sexo masculino $(\mathrm{M}=4,6)$; 'Importância do trabalho em grupo em casos reais de RSO', mais valorizado pelas estudantes do sexo feminino ( $(M=4,9)$ do que pelos estudantes masculinos $(M=4,69)(\mathrm{p}=0,010)$ e 'Briefing da empresa' $(\mathrm{p}=0,04)$, em que as estudantes $(\mathrm{M}=4,29)$ também avaliam melhor do que os estudantes do sexo masculino $(\mathrm{M}=3,97)$

\section{Conclusões}

Este artigo analisou o caso de uma disciplina específica - Comportamento Organizacional - que inclui um módulo sobre Responsabilidade Social das Organizações. A opção pela escolha de uma UC transdisciplinar, lecionada por uma socióloga, focou-se na prática pedagógica que se considerou poder ser interessante para partilha e não em conteúdos teóricos especificamente de Sociologia.

Da avaliação efetuada em dois momentos distintos, concluiu-se que o nível de conhecimento dos estudantes sobre RSO anterior à experiência de ensino era, em geral, bastante baixo. Os estudantes revelaram mais associações com as dimensões sociais externas e ambientais da RSO. Porém, ao analisar os mapas cognitivos, foi possível reconhecer que o leque de palavras associadas a cada uma das dimensões foi bastante redutor, mesmo na dimensão ambiental, contrariando diversos estudos segundo os quais a geração $\mathrm{Z}$ apresentaria maior nível de preocupação ambiental (Finisterra do Paço e Raposo, 2019).

$\mathrm{O}$ enfoque da UC foi propositadamente centrado nas questões de responsabilidade social nos contextos organizacionais, pelo que o efeito formação revelou-se maior nas questões relacionadas com estes aspetos e menos evidente nas questões relacionadas com as responsabilidades sociais pessoais. Em relação ao comportamento dos estudantes como cidadãos socialmente responsáveis, apesar da variação positiva verificada, esperava-se que a gama de comportamentos identificados como socialmente 
responsáveis fosse maior, o que não aconteceu. Esta conclusão dá pistas para futuras edições do módulo de RSO, onde os comportamentos socialmente responsáveis dos cidadãos devem ser mais abordados e discutidos.

A avaliação feita pelos estudantes a esta UC no final do semestre, no inquérito de avaliação desenvolvido pela instituição de ensino, foi bastante positiva: avaliação global da UC: 9,6 (em 10). A avaliação dos métodos pedagógicos foi de 9,33 (em 10). O que denota a satisfação geral dos estudantes quanto a esta metodologia pedagógica e processo de aprendizagem dos conteúdos ministrados.

No que diz respeito à avaliação dos estudantes sobre as diferentes experiências de ensino, a possibilidade de trabalhar com casos reais, é, sem dúvida, um fator motivador para a aprendizagem. A metodologia de aprendizagem de resolução de problemas deve ser reforçada no ensino de conteúdos relacionados com a RSO (graduação ou pós-graduação).

\section{Referências bibliográficas}

BAECKER, Dirk - A sociology of management in management education. In STEYAERT, Chris; BEYES, Timon, PAR KER, Martin (eds.) - The Routledge Companion to Reinventing Management Education. London: Routledge, 2016. ISBN: 9780415727372. p. 107-120.

BLANCO, Antonio - Las aportaciones de la sociología española a la responsabilidad social empresarial. Revista Internacional de Sociología [em linha]. 68: 3 (2010), p. 577-602 [consult. 21 mar. 2021]. Disponível em: https://revintsociologia.revistas.csic.es/index.php/revintsociologia/article/view/345/352. ISSN : 1988-429X.

BRIN KMANN, Johannes - The potential use of sociological perspectives for business ethics teaching. Journal of Business Ethics [em linha]. 156: 1 (2019), p. 273-287 [consult. 21 mar. 2021]. Disponível em:

https://link.springer.com/content/pdf/10.1007/s10551-017-3636-x.pdf. ISSN : 1573-0697.

CASTELO, Branco Manuel - Economic-Sociological Perspectives on CSR. In: IDOWU, Samuel O.; CAPALDI, Nicholas; ZU, Liangrong; DIAS-GUPTA, Amanda (eds) - Encyclopedia of Corporate Social Responsibility. Berlin: Springer, 2013. ISBN: 978-3-642-28087-0. p. 903-909.

DITOM ASO, Nancy - Sociologists Teaching in Business Schools: Prospects and Opportunities. ClinicalSociologyReview [em linha].4:1 (1986), p. 140-155 [consult. 21 mar.2021]. Disponível em: https://digitalcommons.wayne.edu/cgi/viewcontent .cgi?article $=1080 \&$ contex $\mathrm{t}=\mathrm{csr}$. ISSN : 0730-840X.

DUARTE, F. - Rekindling the sociological imagination as a pedagogical "package" in management education. Journal of Management Education [em linha]. 33: 1 (2009), p. 59-76. [consult. 21 mar. 2021]. Disponível em: https://journals.sagepub.com/doi/ abs/10.1177/1052562908319996. ISSN : 1552-6658.

EGREJA, Catarina - A Sociologia como disciplina em cursos de outras áreas científicas no ensino superior português. CIES e-Working Paper [em linha]. 188 (2014), p. 1-27. [consult. 21 mar. 2021]. Disponível em: https://repositorio.iscte-iul.pt/bitstream/10071/9886/1/CIES-W P188_ Egreja.pdf. ISSN : 1647-0893.

EGREJA, Catarina - O ensino da sociologia em cursos superiores de outras áreas de formação: a perspetiva de docentes e diretores. Sociologia, Problemas e Práticas [em linha] 82 (2016), p. 125-143. [consult. 21 mar. 2021]. Disponível em: https://journals.openedition.org/spp/2534. ISSN : 2182-7907. 
ESTEVES, António Joaquim - Métodos, práticas pedagógicas e públicos da Sociologia: um ponto de vista situado. In GONÇALVES, Carlos Manuel; RODRIGUES, Eduardo; AZEVEDO, Natália (orgs.) - Sociologia no Ensino Superior. Conteúdos, Práticas Pedagógicas $e$ Investigação: Actas do Encontro Realizado nos Dias 6 e 7 de Dezembro de 2002 na FLUP. Porto: Departamento de Sociologia da FLUP, 2004. ISBN : 972-9350-86-8. p. 61-77.

FIN ISTERRA DO PAÇO, Arminda M.; RAPOSO, Mário Lino Barata - Green consumer market segmentation: empirical findings from Portugal. International Journal of Consumer Studies [em linha]. 34: 4 (2010), p. 429-436. [consult. 21 mar. 2021]. Disponível em: https://onlinelibrary.wiley.com/doi/epdf/10.1111/j.1470-6431.2010.00869.x. ISSN : 1470-6431.

GIDDENS, Anthony - As Consequências da Modernidade. Oeiras: Celta, 1992. ISBN: 9789728027919.

GOMES, Sandra - A sociologia no ensino de não sociólogos: relato de uma experiência pedagógica. In GONÇALVES, Carlos Manuel; RODRIGUES, Eduardo; AZEVEDO, Natália (orgs.) - Sociologia no Ensino Superior. Conteúdos, Práticas Pedagógicas e Investigação: Actas do Encontro Realizado nos Dias 6 e 7 de Dezembro de 2002 na FLUP. Porto: Departamento de Sociologia da FLUP, 2004. ISBN: 972-9350-86-8. p. 157-164.

GOMES, Sandra; NOGUEIRA, Mafalda; NEVES, Cidália - Higher Education Institutions as Corporate Social Responsibility drivers: teaching and learning to transform the world. s.1.: IATED, 2020. 10 p. (Edulearn20 Proceedings). Comunicação apresentada na 12th International Conference on Education and New Learning Technologies, conferência em linha, 2020. ISBN : 978-84-09-17979-4.

LAZ, Cheryl - Republication of "Science Fiction and Introductory Sociology: The Handmaid in the Classroom". Teaching Sociology [em linha]. 48: 1 (2020), p. 54-62. [consult. 14 abr. 2021]. Disponível em: https://journals.sagepub.com/doi/pdf/10.1177/0092055X19894639. ISSN : 0092055X.

LOES, Chad N.; PASCARELLA, Ernest T. - Collaborative learning and critical thinking: Testing the link. The Journal of Higher Education [em linha]. 88: 5 (2017), p. 726-753. [consult. 14 abr. 2021]. Disponível em: https://www.tandfonline.com/doi/pdf/10.1080/00221546.2017.1 291257 ?need Access=true. ISSN : 1538-4640.

LOURDEL, Nathalie; GRONDRAN, Natacha; LAFOREST, Valérie; DEBRAY Bruno; BRODHAG, Christian - Sustainable Development cognitive map: a new method of evaluating student understanding. [em linha]. International Journal of Sustainability in Higher Education 2: 8 (2007), p. 170-182. [consult. 14 abr. 2021]. Disponível em: https://citeseerx.ist. psu.edu/viewdoc/download?doi=10.1.1.662.4742\& rep=rep1\& type=pdf. ISSN : 1467-6370.

MAYBERRY, Maralee - Reproductive and resistant pedagogies: The comparative roles of collaborative learning and feminist pedagogy in science education. Journal of Research in Science Teaching [em linha]. 35: 4 (1998), p. 443-459. [consult. 14 abr. 2021]. Disponível em: https://onlinelibrary.wiley.com/doi/epdf/10.1002/\% 28SICI\% 291098$-2736 \% 28199804 \% 2935 \% 3$ A $4 \% 3$ C $443 \% 3$ A\% 3 AAID-TEA $14 \% 3$ E3 .0.CO \% 3 B2 -A. ISSN :1098-2736.

O'DONNELL, Angela M., HMELO-SILVER, Cindy E. - Introduction what is collaborative learning?: An overview. In HMELO-SILVER, Cindy; CHINN, Clark; CHAN, Carol; O'DON NELL, Angela (eds.) - The international handbook of collaborative learning. New York: Routledge, 2013. ISBN: 9780203837290. p. 13-28.

PEDERSEN, Daphne E. - Active and Collaborative Learning in an Undergraduate Sociological Theory Course. Teaching Sociology [em linha]. 38: 3 (2010), p. 197-206. [consult. 14 abr. 2021]. Disponível em: https://journals.sagepub.com/doi/pdf/10.1177/0092055X10370119. ISSN : $0092055 \mathrm{X}$.

RENDTORFF, Jacob Dahl - An Interactive Method for Teaching Business Ethics, Stakeholder Management and Corporate Social Responsibility (CSR). Journal of Business Ethics 
Education [em linha]. 12 (2015), p. 93-106. [consult. 14 abr. 2021]. Disponível em: https:// doi.org/10.5840/jbee2015126. ISSN : 2044-4559.

TOLICH, Martin; CHOE, Louisa; DOESBURG, Adam; FOSTER, Amy; SHAW, Rachel; WITHER, David - Teaching research ethics as active learning: Reading Venkatesh and Goffman as curriculum resources. International Journal of Social Research Methodology [em linha]. 20: 3 (2017), p. 243-253. [consult. 14 abr. 2021]. Disponível em: https://www.tandfonline.com/doi/pdf/10.1080/13645579.2017.1287870?need Access=true. ISSN : 1464-5300.

YIN, Yue; VANIDES, Jim; RUIZ-PRIMO, Maria Araceli; AYALA, Carlos C.; SH AVELSON, Richard J. - Comparison of two concept-mapping techniques: Implications for scoring, interpretation, and use. Journal of Research in Science Teaching [em linha]. 42: 2 (2005), p. 166-184. [consult. 14 abr. 2021]. Disponível em: https://onlinelibrary.wiley.com/doi/ epdf/10.1002/tea.20049. ISSN:1098-2736.

UNITED NATIONS - Transforming our world: the 2030 Agenda for Sustainable Development [em linha]. United Nations. S.1.: 2015. [consult. 14 abr. 2021]. Disponível em: https://sdgs. un.org/publications/transforming-our-world-2030-agenda-sustainable-development-17981.

VIDAL, Rodolphe - L'intervention des sciences sociales dans le champ de la responsabilité sociale des entreprises. Tracés. Revue de Sciences Humaines [em linha]. 10 (2010), p. 65-76. [consult. 14 abr. 2021]. Disponível em: https://journals.openedition.org/traces/4693. ISSN: 1963-1812.

- Receção: 16.06 .2021

- Aprovação: 17.10.2021 